ARTICLE

\title{
Global population structure and genotyping framework for genomic surveillance of the major dysentery pathogen, Shigella sonnei
}

\author{
Jane Hawkey (1,14凶, Kalani Paranagama ${ }^{1,14}$, Kate S. Baker ${ }^{2}$, Rebecca J. Bengtsson ${ }^{2}$, François-Xavier Weill (0 ${ }^{3}$, \\ Nicholas R. Thomson (10 4,5, Stephen Baker (1) 6,7, Louise Cerdeira (10 1, Zamin Iqbal (1) ${ }^{8}$, Martin Hunt ${ }^{8,9}$, \\ Danielle J. Ingle $e^{10,11}$, Timothy J. Dallman ${ }^{12}$, Claire Jenkins ${ }^{12}$, Deborah A. Williamson (i) ${ }^{10,13}$ \& Kathryn E. Holt (iD) 1,5
}

Shigella sonnei is the most common agent of shigellosis in high-income countries, and causes a significant disease burden in low- and middle-income countries. Antimicrobial resistance is increasingly common in all settings. Whole genome sequencing (WGS) is increasingly utilised for S. sonnei outbreak investigation and surveillance, but comparison of data between studies and labs is challenging. Here, we present a genomic framework and genotyping scheme for S. sonnei to efficiently identify genotype and resistance determinants from WGS data. The scheme is implemented in the software package Mykrobe and tested on thousands of genomes. Applying this approach to analyse $>4,000$ S. sonnei isolates sequenced in public health labs in three countries identified several common genotypes associated with increased rates of ciprofloxacin resistance and azithromycin resistance, confirming intercontinental spread of highly-resistant S. sonnei clones and demonstrating the genomic framework can facilitate monitoring the spread of resistant clones, including those that have recently emerged, at local and global scales.

\footnotetext{
${ }^{1}$ Department of Infectious Diseases, Central Clinical School, Monash University, Melbourne, VIC, Australia. ${ }^{2}$ Department of Clinical Infection, Microbiology, and Immunology, Institute for Infection, Ecological and Veterinary Sciences, University of Liverpool, Liverpool, UK. ${ }^{3}$ Institut Pasteur, Unité des bactéries pathogènes entériques, Paris, France. ${ }^{4}$ Wellcome Sanger Institute, Wellcome Genome Campus, Hinxton, UK. ${ }^{5}$ Dept Infection Biology, London School of Hygiene \& Tropical Medicine, London, UK. ${ }^{6}$ University of Cambridge School of Clinical Medicine, Cambridge Biomedical Campus, Cambridge, UK. 7 Department of Medicine, University of Cambridge School of Clinical Medicine, Cambridge Biomedical Campus, Cambridge, UK. ${ }^{8}$ European Molecular Biology Laboratory-European Bioinformatics Institute, Hinxton, UK. ${ }^{9}$ Nuffield Department of Medicine, University of Oxford, Oxford, UK. 10 Microbiological Diagnostic Unit Public Health Laboratory, Department of Microbiology and Immunology at the Peter Doherty Institute for Infection and Immunity, The University of Melbourne, Melbourne, VIC, Australia. ${ }^{11}$ Research School of Population Health, Australian National University, Canberra, ACT, Australia. ${ }^{12}$ National Infection Service, Public Health England, London, UK. ${ }^{13}$ Department of Microbiology, Royal Melbourne Hospital, Melbourne, VIC, Australia.

${ }^{14}$ These authors contributed equally: Jane Hawkey, Kalani Paranagama. ${ }^{凶}$ email: jane.hawkey@monash.edu
} 
S higella spp are Gram-negative bacterial pathogens that cause shigellosis (bacterial dysentery). Shigella are transmitted via the faecal-oral route and estimated to cause $\sim 188$ million infections annually, leading to $\sim 160,000$ deaths mainly in young children ${ }^{1}$. In low- and middle-income settings, most of the Shigella disease burden of shigellosis is in children under five years ${ }^{2}$, however in high-income countries Shigella is frequently detected in returned travellers or men who have sex with men (MSM) $)^{3,4}$. Shigella sonnei is the most frequently isolated agent of shigellosis in high-income countries and in those that are economically developing ${ }^{1,5,6}$. S. sonnei emerged recently $(\sim 350$ years $\left.\mathrm{ago}^{7}\right)$, share a single serotype, and display limited genomic diversity (all belong to ST152 complex by multi-locus sequence typing (MLST)). These properties make it difficult to differentiate and track $S$. sonnei strains ${ }^{7}$, motivating adoption of whole-genome sequencing (WGS) for research and public health surveillance of this organism ${ }^{8}$. Core-genome MLST (cgMLST) is available via the Escherichia coli scheme in EnteroBase ${ }^{9}$ but has not been widely adopted for $S$. sonnei surveillance, and most public health labs and research studies rely on the higher-resolution technique of single nucleotide variant (SNV)-based phylogenetics analysis.

The global population of $S$. sonnei is divided into five major lineages ${ }^{7,10}$. Several WGS studies have investigated regional $S$. sonnei epidemiology and population structure, including in Asia $^{11-14}$, Australia ${ }^{4}$, the Middle East ${ }^{15}$, South America ${ }^{10}$, and the United Kingdom ${ }^{16-18}$; and defined additional sub-lineagelevel phylogenetic groups of local epidemiological importance, associated with features such as ciprofloxacin-resistance ${ }^{19}$, transmission within Orthodox Jewish communities ${ }^{15}$, or transmission amongst $\mathrm{MSM}^{4,17}$. S. sonnei from Asia, Europe, Australia and North America have for $>20$ years been dominated by Lineage 3 strains that are resistant to early first-line antimicrobials (trimethoprim-sulfamethoxazole, tetracycline, and streptomycin) due to the presence of antimicrobial resistance (AMR) genes acquired horizontally via the small plasmid spA and a chromosomal Tn7-like transposon ${ }^{7,20,21}$. Resistance to chloramphenicol and/or ampicillin is also observed (e.g., via acquisition of the Shigella resistance locus (SRL) $\left.{ }^{10,22}\right)$. Reduced susceptibility to fluoroquinolones has emerged on multiple occasions and in multiple locations through acquisition of point mutations within the quinolone resistance determining region (QRDR) of $g y r A^{7,11}$. Resistance to ciprofloxacin has emerged at least once via the accumulation of three QRDR mutations (2 in gyrA and one in $\operatorname{parC}$ ) in a South Asian sublineage that has since been detected on multiple continents ${ }^{12-14,23}$. Resistance to the last few remaining drugs is increasing through the acquisition and maintenance of plasmids carrying $m p h(A)$ and ermB (azithromycin resistance) or extended-spectrum beta-lactamase (ESBL) genes (ceftriaxone resistance), often in combination with additional aminoglycoside resistance genes ${ }^{11,14,16,18,24}$.

In many countries, $S$. sonnei is a notifiable infection and subject to public health surveillance and outbreak investigations, which are increasingly conducted using $\mathrm{WGS}^{8,25-28}$. However, the lack of a defined global genomic framework and accompanying genotype nomenclature hampers both local reporting, outbreak detection, and patterns of spread within regions. For example, most $S$. sonnei WGS studies have reported which of the five major lineages their novel isolates belong to, but have had to download public reference genome data, construct whole genome alignments, and infer phylogenies to achieve this basic identification ${ }^{23,27}$. Studies of MSM S. sonnei in different settings have designated different names for the same lineages $4,16,18$, obscuring the fact that the same clones are spreading amongst MSM communities in different countries, and the only way to recognize this currently is through construction of whole-genome phylogenies incorporating data from multiple prior studies ${ }^{29}$.
WGS-based genotyping frameworks based on single nucleotide variants (SNVs) have been widely adopted for the bacterial pathogens Mycobacterium tuberculosis ${ }^{30}$ and Salmonella enterica serovar Typhi ${ }^{31}$, which display similarly low levels of genomic diversity to $S$. sonnei. These frameworks enable fast and accurate typing of clinical isolates from WGS data without the need for time-consuming comparative genomics or phylogenetics, facilitating straightforward identification of (and cross-jurisdictional communication about) epidemiologically important lineages from WGS data.

Here, we describe the global population structure for S. sonnei and (i) propose a hierarchical SNV-based genotyping scheme, which we define using 1935 globally distributed genomes; (ii) implement the scheme within the free and open-source Mykrobe $^{32}$ software alongside detection of genetic determinants that are highly predictive of fluoroquinolone susceptibility phenotypes in S. sonnei ${ }^{28}$; and (iii) validate this approach to genotyping using an additional 2015 genomes that were sequenced in public health laboratories and deposited in the publicly available GenomeTrakr database. Code is available at https://github.com/ katholt/sonneityping. By applying this novel genotyping framework to $S$. sonnei WGS data generated in public health laboratories on three continents, we demonstrate the utility of the new scheme for tracking and reporting emerging AMR clones both within and between jurisdictions.

\section{Results}

Defining phylogenetically informative genotypes for S. sonnei. In order to define the global population structure and identify clades and marker SNVs, we collated 1935 publicly available $S$. sonnei genomes from eight studies $4,7,10-13,15,16$ as our "discovery" dataset (see Supplementary Table 1). These genomes represent isolates from 48 countries, collected between 1943 and 2018 (Fig. 1c, d, Table 1). The majority originate from Asia (32.4\%), Europe $(29.3 \%)$, Australia (18.8\%), or Latin America and the Caribbean (17.4\%) (Table 1). The data set is diverse in terms of acquired AMR genes (median 9 per genome, range 0-21), and includes $150(7.8 \%)$ genomes known to be associated with MSM.

The recombination-filtered core-genome maximum likelihood phylogeny inferred from these genomes (Fig. 1b) was robust (median bootstrap support 100\%) and exhibited the five previously-described deep branching lineages ${ }^{7,10}$. Lineage 3 was most common (86.9\%), followed by Lineage $2(10.7 \%)$, Lineage 5 $(1.4 \%)$, Lineage $1(0.9 \%)$ and Lineage $4(n=1)$. The pairwise core-genome SNV distance distribution revealed peaks and troughs which we used to set thresholds to define clusters at different levels of resolution (Fig. 2a). A threshold of 600 pairwise SNVs separated the five major lineages; troughs at 215 SNVs and 100 SNVs were used to define higher-resolution genetic clusters. (A similar structure was recovered using hierarchical Bayesian clustering of the SNV matrix using FastBAPS, but with less consistent levels of divergence between clusters; see Supplementary Fig. 1). Mapping the pairwise SNV threshold-defined clusters onto the phylogeny confirmed that each cluster corresponded to a monophyletic group with $100 \%$ bootstrap support, which we designate as clades ( $n=29$, using $215-\mathrm{SNV}$ threshold) and subclades ( $n=96$, using $100-\mathrm{SNV}$ thresholds).

We used these cluster memberships to define hierarchical genotypes with nomenclature in the form [lineage].[clade]. [subclade] (see Fig. 1a). Similar to the M. tuberculosis and S. Typhi schemes, this hierarchical nomenclature facilitates easy recognition of relationships between genotypes; e.g., subclades 3.6.1, 3.6.2, ..., 3.6. $\mathrm{N}$ are sister groups in the whole-genome phylogeny, nested within clade 3.6, which falls within Lineage 3 (see Fig. 1a). The median pairwise distance between genomes of 


\section{a}

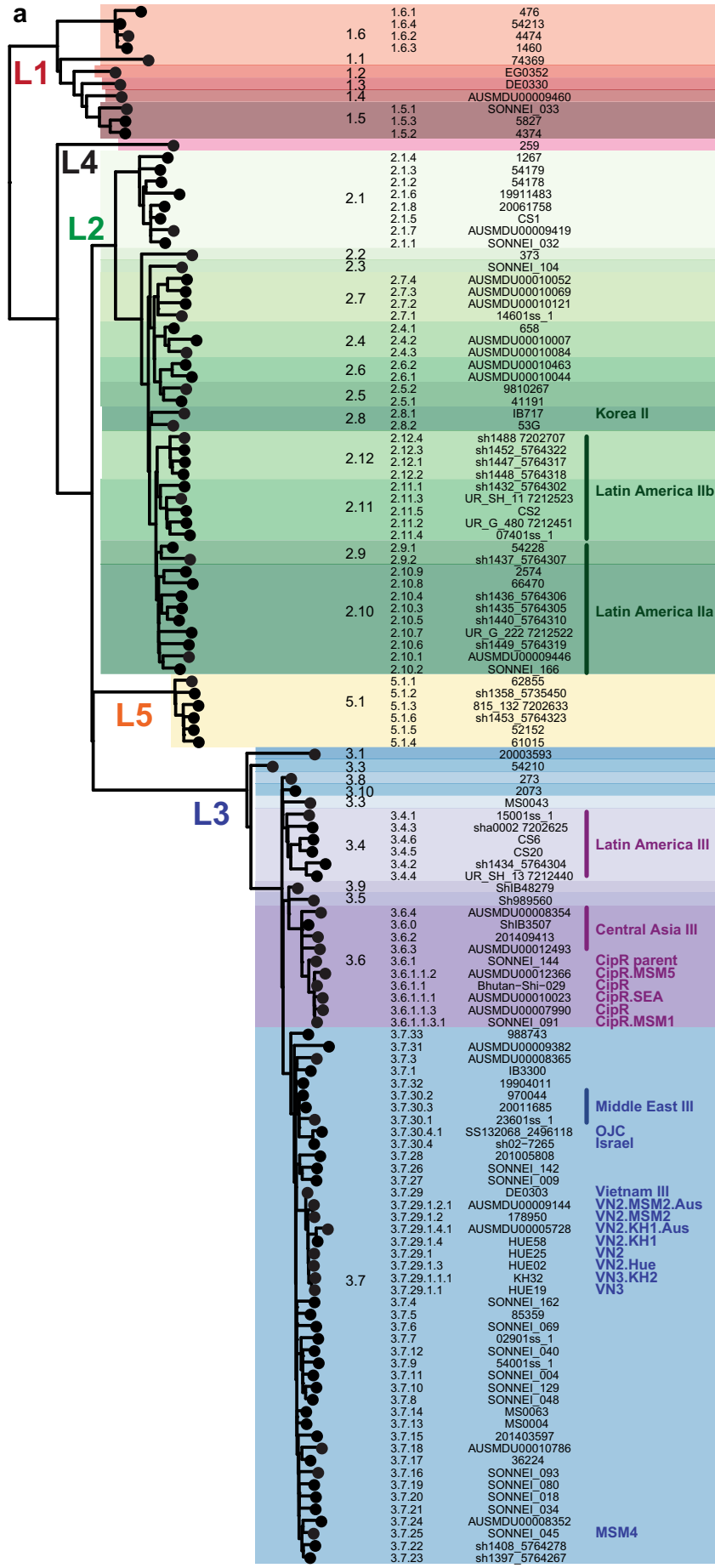

b

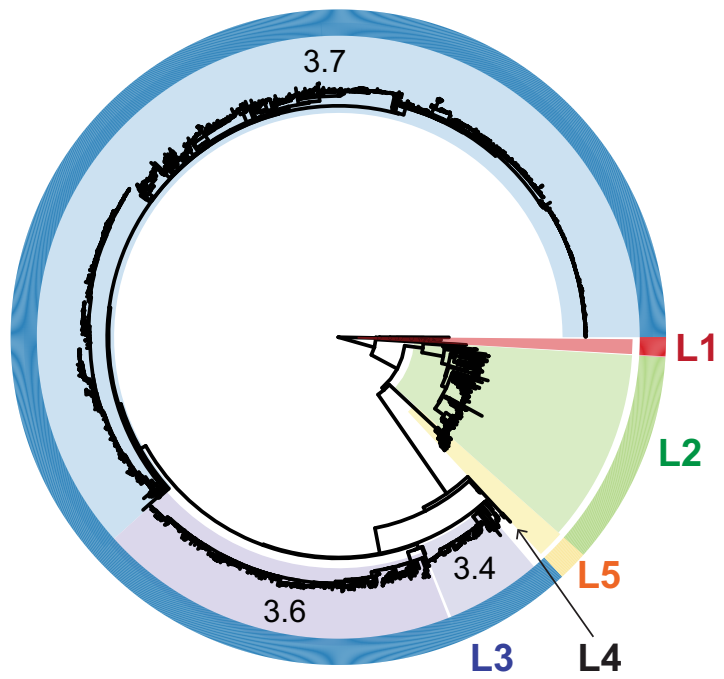

C

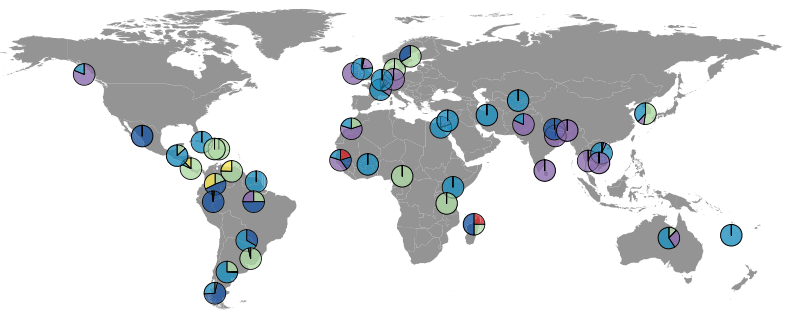

$\begin{array}{lllllll}1 & 2 & 3 & 3.6 & 3.7 & 4 & 5\end{array}$

Lineage/Clade $\square \square \square \square \square \square \square$

d

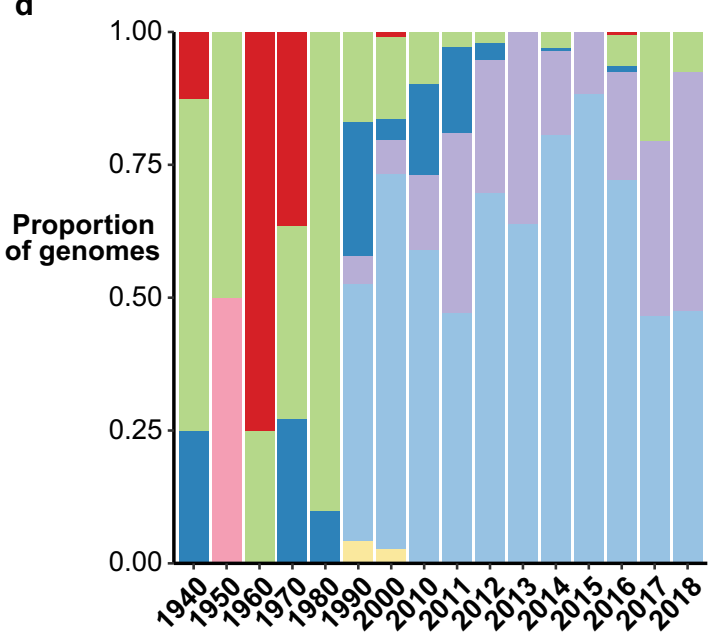

Fig. 1 Population structure, temporal distribution and geographic distribution of the 1935 S. sonnei genomes in the discovery set. a Maximum likelihood phylogeny (outgroup rooted using $E$. coli) of one representative per genotype. Lineages are labelled LX, where $X$ is the lineage number. Highlighting and column 1 indicate clades, column 2 indicates genotype, column 3 shows strain names, column 4 shows human readable genotype names (for epidemiological groups noted in Table 2). b Maximum likelihood phylogeny (outgroup rooted using E. coli), c frequencies by geographic region, and d frequencies by decade/year; for all discovery set genomes and coloured by lineage and major clades (3.6, 3.7, see inset legend). Interactive version of linked phylogeny, map and timeline for this data set are available online in Microreact (https://microreact.org/project/fG2N7huk9oZNCaVHu8rukr).

the same clade or subclade was 138 or 47 core-genome SNVs, respectively (Fig. 2b).

Whilst the discovery set is not a systematic sampling across geographic regions, it can provide some preliminary insights into the global distribution of $S$. sonnei genotypes. Lineages were broadly distributed across continents (with the exception of Lineage 5 and the singleton Lineage 4, see Fig. 1c, Supplementary
Data 1), however the majority of clades $(n=24,83 \%)$ were represented by isolates from just one $(n=16,57 \%)$ or two $(n=7$, $23 \%$ ) continents. At the other extreme, clades 3.6 and 3.7 were widely distributed, with representatives on all six continents (Fig. 1b). Clades 3.6 and 3.7 were the most common overall (19\% and $62 \%$, respectively) and accounted for the majority of S. sonnei from all continents except Latin America, where clades 2.10, 2.11, 
Table 1 Geotemporal distribution of 1935 S. sonnei genomes in the discovery dataset.

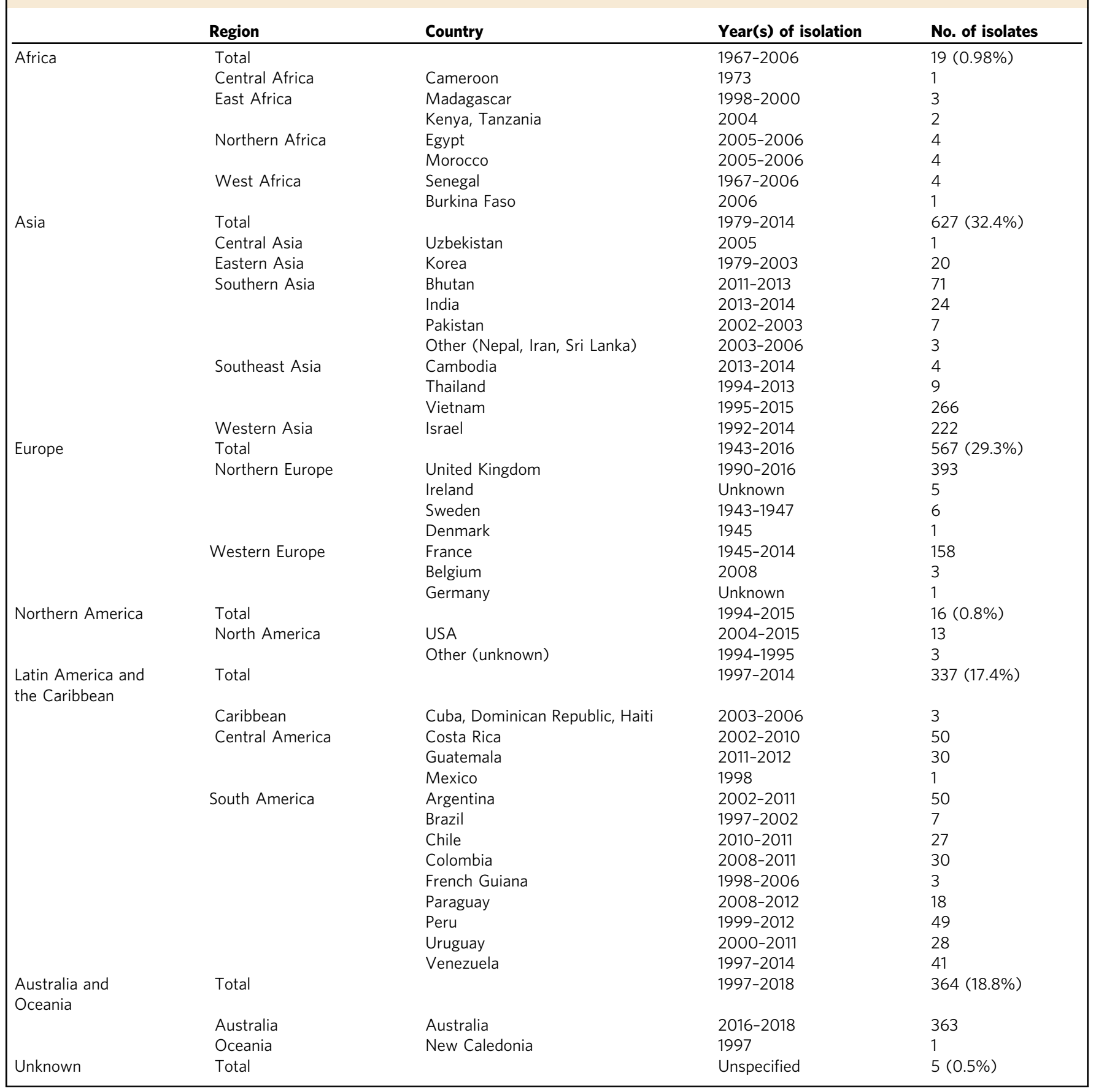

$2.12,3.4,3.6$ and 5.1 were common (8-28\% each). Subclades showed even greater geographic specificity, with $74 \%(n=71)$ represented by a single continent only and $72 \% \quad(n=69)$ represented by a single region only. Fifty-nine subclades $(61 \%)$ were dominated by genomes from a single country.

As a key goal of the $S$. sonnei genotyping scheme is to facilitate identification and communication about subtypes of public health interest, we reviewed the position of genetic clusters that have been described in the literature as being of epidemiological importance (Table 2). Groups previously identified as being associated with specific geographical regions mapped mainly to clades or subclades defined in the genotyping scheme (Table 2). Most groups previously defined on the basis of AMR or transmission patterns comprised more recently-emerged clusters, forming monophyletic groups within our subclade-level genotypes. Hence we created additional higher-resolution genotypes nested within subclades to demarcate these groups (e.g., 3.6.1.1, 3.6.1.1.1; see Table 2), and anticipate adding more genotypes as new resistant groups emerge in future. For example, the ciprofloxacin resistant triple-mutant sublineage ${ }^{12-14}$ comprised a monophyletic group within subclade 3.6.1 that we define as genotype 3.6.1.1; distinct subgroups within this have also been described, associated with South East Asia (genotype 3.6.1.1.1), and MSM communities in Australia (3.6.1.1.2) or the UK (3.6.1.1.3.1) (see Table 2).

To facilitate communication about genotypes of epidemiological interest, we also assigned them human readable aliases (e.g., 3.6.1.1 = CipR, 3.6.1.1.1 = CipR.SEA, 3.6.1.1.2 = CipR.MSM5, 
a

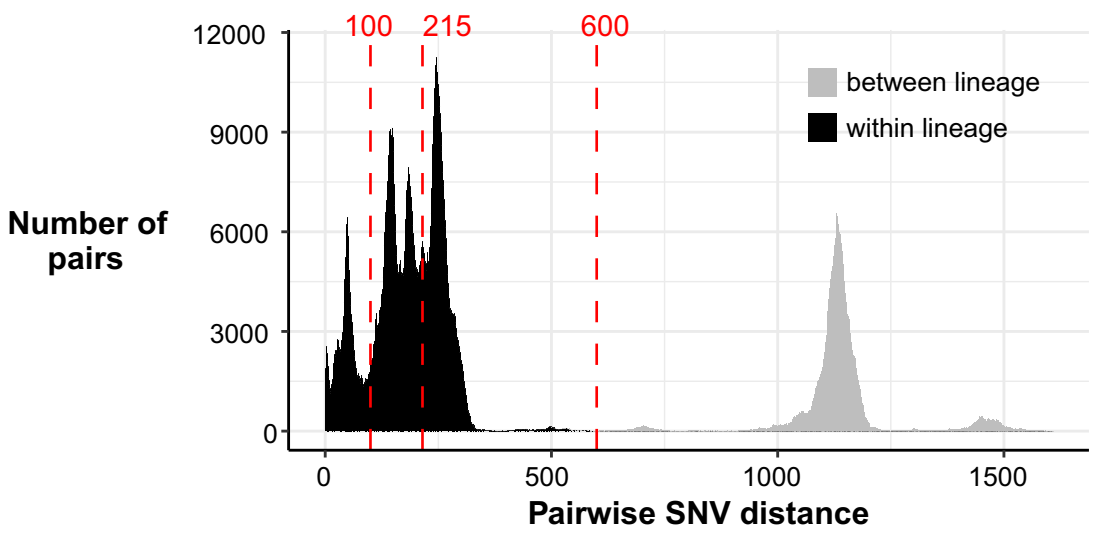

b

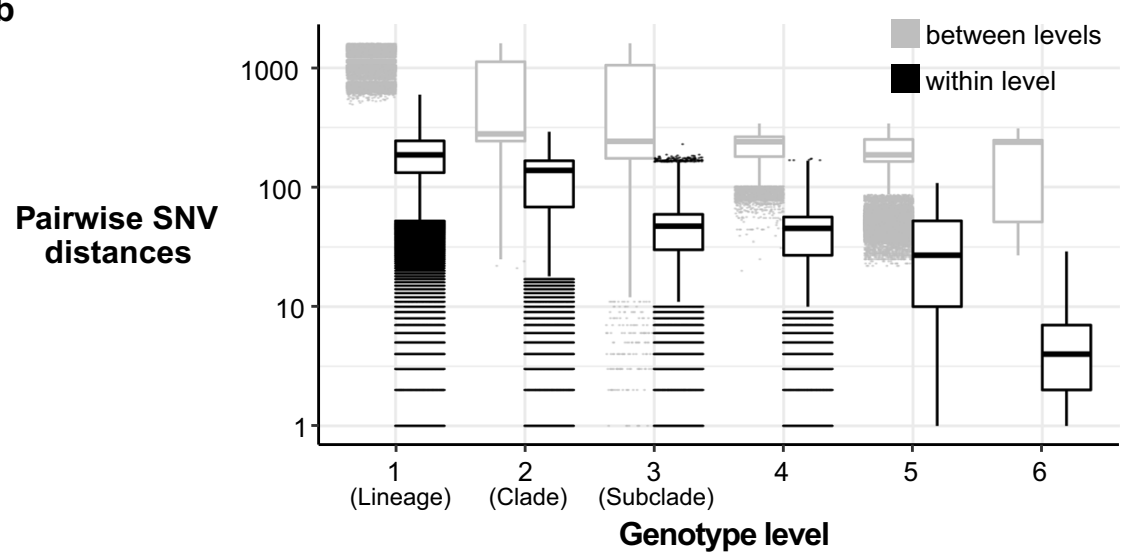

Fig. 2 Distribution of SNV distances in discovery genomes. a Histogram of pairwise SNV distances between all discovery genomes, coloured by lineage comparison as per legend. Red lines mark SNV cut-offs used to define lineage, clade and subclade levels in genotyping scheme. b Boxplots of pairwise SNV distances (log scale, $n=1,873,081$ pairwise distances) between discovery genomes at different hierarchical levels of the defined genotyping scheme. Boxes indicate the median (bold line), 25th to 75th percentiles (box), and the 5th and 95th percentile (whiskers), with outliers shown as points. Lineage, Clade and Subclade refer to the first three levels of the scheme. ' 4 ' indicates the fourth level of the scheme (i.e., the final ' 1 ' in 3.6.1.1), ' 5 ' the fifth level (i.e., the ' 1 ' in 3.7.30.4.1), and ' 6 ' the sixth level (i.e., the final ' 1 ' in 3.6.1.1.3.1).

see Table 2). As far as possible these aliases map to names given in previous publications, e.g., the MSM clade numbers designated in ${ }^{16,17}$. Most of the epidemiological groups of interest belong to Lineage 3, and detailed phylogenies for these groups are provided in Supplementary Fig. 2. In addition to being monophyletic on the tree, these higher-level genotypes were supported by FastBAPS analysis (Supplementary Fig. 1). Pairwise distances within and between genotypes of all levels are shown in Fig. $2 \mathrm{~b}$.

Development and validation of SNV-based scheme for assigning genotypes. We identified marker SNVs unique to each genotype (147 SNVs in total, see Supplementary Data 2) and implemented code to assign new genomes to genotypes based on presence of these markers (see "Methods"). To validate this approach, we downloaded and genotyped 2015 additional $S$. sonnei genomes from GenomeTrakr (referred to as validation set, see Supplementary Data 1). These genomes originate from public health laboratories in three countries $(n=609$ USA, $n=1325$ UK, $n=11$ Israel, $n=70$ country unknown), with isolation dates between 2015 and 2019 .

We identified 17 different genotypes, all belonging to clades 3.6 or 3.7 (Supplementary Table 2). The vast majority $(70 \%, n=$ 1403) belonged to clade 3.6. Genotype 3.6.1.1.2 (CipR.MSM5) was the most prevalent, assigned to $26.6 \%$ of the genomes, followed by 3.6.1.1 (CipR, 19.6\%) (Supplementary Table 2). The UK GenomeTrakr genomes yielded the greatest number of genotypes $(n=16)$, followed by the USA $(n=13)$; likely due to a high number of travel-associated cases. All GenomeTrakr genomes deposited from Israel were identified as 3.7.30.4 (Israel III, 9\%) or 3.7.30.4.1 (OJC, 91\%); these genotypes were also detected amongst UK and USA genomes.

To verify the genotyping scheme accurately captured the population structure present in the GenomeTrakr isolates, we constructed a core-genome phylogeny including both the validation set and Lineage 3 discovery set (total $n=3696$ genomes, see "Methods") and mapped the genotype assignments to this tree (see Supplementary Fig. 3 and Microreact https://microreact.org/project/g8BvA2JCXWaZNDyPyjsWXF).

All groups of isolates sharing a genotype assignment based on marker SNVs constituted monophyletic clades within the coregenome phylogeny, consistent with the expected behaviour of the scheme. This was true for all levels in the hierarchical scheme, including clades, subclades, and higher-resolution epidemiological groups.

Distribution of antimicrobial resistance determinants amongst S. sonnei genotypes. We used the genotyping scheme to facilitate exploration of the distribution of AMR determinants in the global S. sonnei population, by assessing the frequency of AMR genes and QRDR SNVs across genotypes (Fig. 3). For this analysis we included $n=6715$ genomes: $n=1935$ discovery set, $n=2015$ validation set and a further $n=2765$ public genomes (accessions listed in Supplementary Data 1). Most AMR determinants were 
Table 2 Details of epidemiological clusters defined within the S. sonnei population.

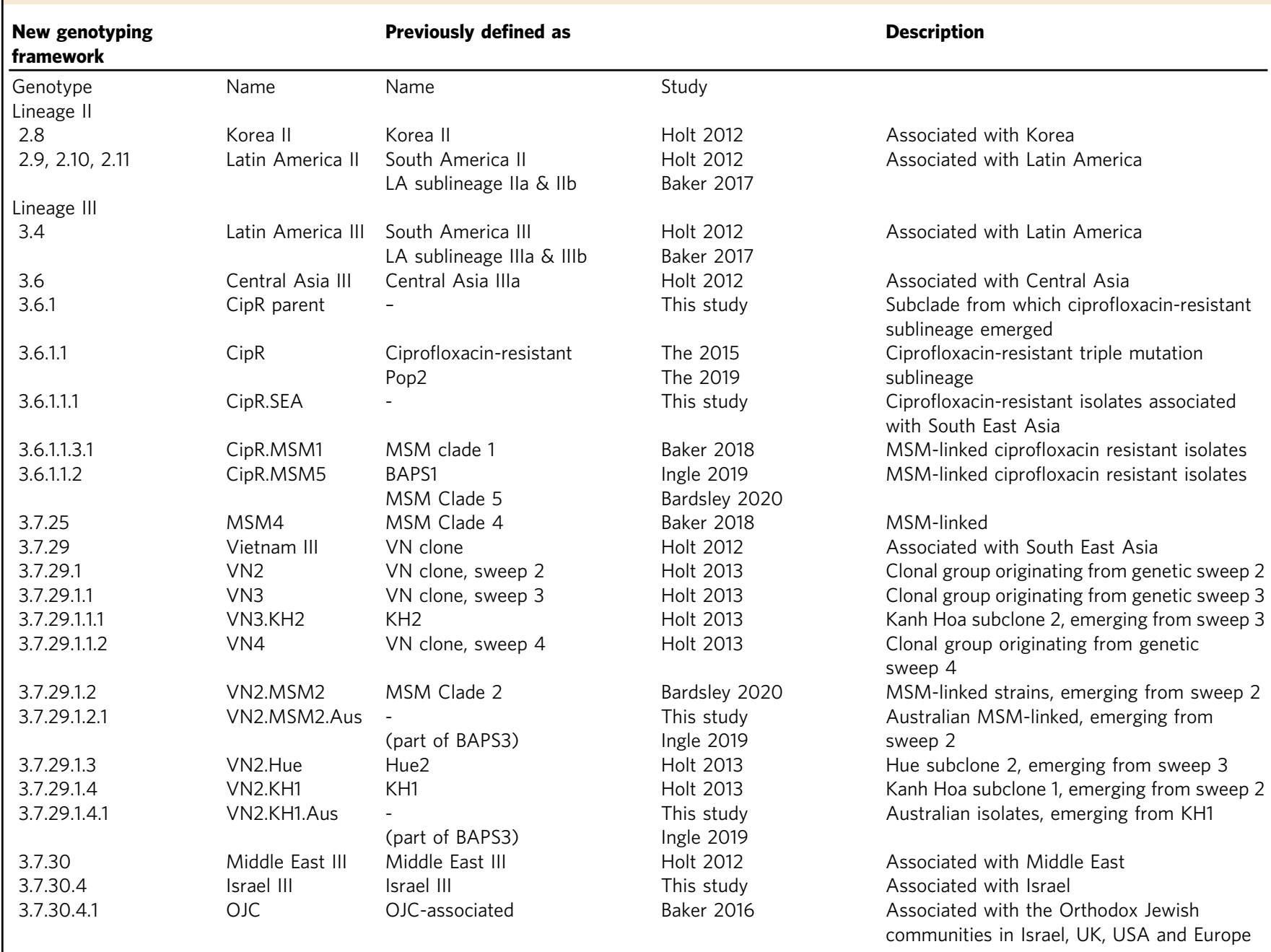

associated with specific genotypes, present amongst either all or no members of each genotype (Supplementary Fig. 4).

Genes conferring resistance to first-line drugs were found in all lineages. Those associated with the spA plasmid $(\operatorname{sul} 2, \operatorname{tet} A(A))$ were found in all lineages but were most widely distributed across clades of Lineage 3 (found in all clades) followed by Lineage 2 (81\% of clades) (Fig. 3, Supplementary Fig. 4). Tn7 transposon genes (tns $A B C D E)$, the class II integron integrase (intA2) and AMR genes in the integron cassette (satA2, dfrA1, aadA1) were absent from Lineage 1 but found in Lineages 2 and 3 (40-41\% of clades) and the single Lineage 5 clade (Fig. 3). This combination of markers for the chromosomally integrated MDR transposon Tn7 was most common in clades 3.6 (99\%), 3.7 (99\%) and 2.12 (88\%), where it was typically accompanied by spA genes (sul2, tet $A(A)$ ) resulting in resistance to co-trimoxazole (Fig. 3). Firstline AMR genes aadA1, tetA(B), catA 1 and $b l a_{\text {OxA-1 }}$, which are known to mobilise together on the SRL, co-occurred in clades 3.3, 3.4 and 3.5, consistent with prior reports of SRL in clade Latin America IIIa (3.4) ${ }^{10}$. Acquired genes $b l a_{\mathrm{TEM}-1}$ and sull were also found at low frequencies across diverse genotypes (Supplementary Fig. 4), suggesting occasional acquisition via mobile elements.

QRDR SNVs were detected in $50.4 \%$ of all genomes, distributed across six clades and 12 subclades (Fig. 3), consistent with frequent emergence of these mutations under selection from drug exposure. Most common was GyrA-S83L (42\% of genomes, six clades, 12 subclades) followed by GyrA-D87G $(30.8 \%$ of genomes, five clades, ten subclades). Single mutants were most common (18.7\% of genomes, five clades, 6 subclades) but double mutants were also observed in 3.6.1 $(n=61 / 6715$ genomes had GyrA-S83L+GyrA-D87G). QRDR triple mutants, associated with ciprofloxacin resistance, were detected only in the CipR sublineage (genotype 3.6.1.1) which harbours GyrA-S83L+GyrAD87G+ParC-S80I. The emergence and evolutionary dynamics of this CipR sublineage from within the Central Asia IIIa clade (genotype 3.6) were recently described in a detailed phylodynamic study of fluoroquinolone resistant isolates from diverse sources by The et al. ${ }^{14}$. That study divided the Central Asia III clade into two populations: Pop1, with either GyrA-D87Y or GyrA-S83L arising on two independent occasions in South Asia in the mid-1990 s; and Pop2, which arose from Pop1 genomes carrying GyrA-S83L in South Asia in the early 2000s, and then acquired GyrA-D87G and ParC-S80I to become fluoroquinolone resistant before spreading geographically ${ }^{14}$. Applying our new genotyping scheme to the genomes from The et al. ${ }^{14}$ (Supplementary Fig. 5), we confirm that Pop1 maps to clade $3.6(n=18)$ and its subclades 3.6.1, 3.6.2, 3.6.3, 3.6.4; and Pop2 maps to sublineage 3.6.1.1 (CipR, $n=239$ ) including its subgroups 3.6.1.1.1 (CipR.SEA, $n=30$ ), 3.6.1.1.2 (CipR.MSM5, $n=3)$ 3.6.1.1.3 $(n=16)$ and 3.6.1.1.3.1 (CipR. MSM1, $n=19$ ).

Determinants of resistance to azithromycin and extendedspectrum cephalosporins were rare and concentrated mainly in clades 3.6 and 3.7. The plasmid-borne azithromycin resistance 


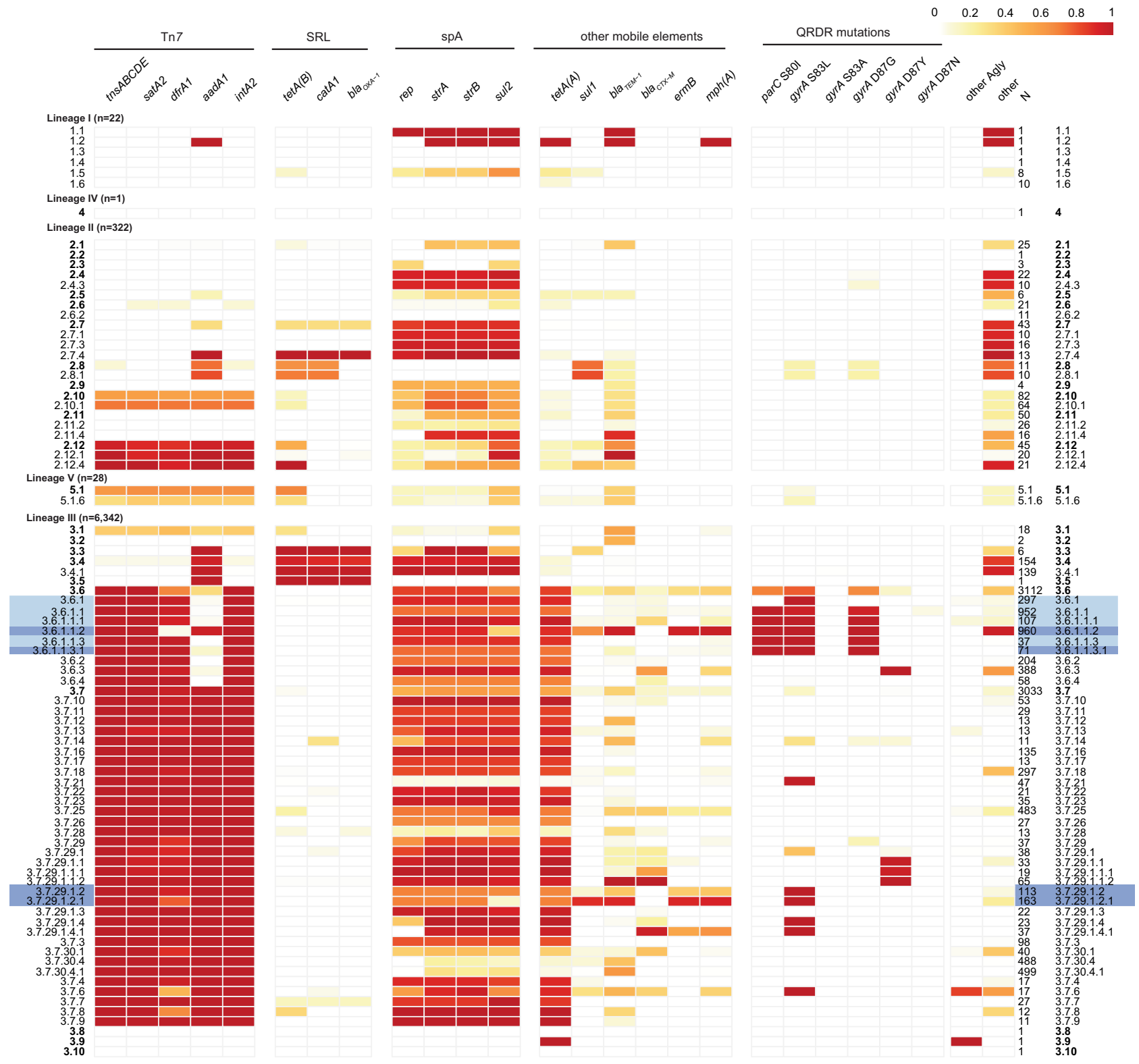

Fig. 3 Frequencies of AMR genetic determinants within individual S. sonnei genotypes, calculated across $\mathbf{6 7 1 5}$ genomes. Cells indicate absence (white) or presence (coloured by proportion as per legend) of each AMR determinant (columns) within each clade or higher-resolution genotype (rows). All clades are included as rows (bold labels); subclades and higher-resolution genotypes represented by $\geq 10$ genomes are also included as distinct rows; number of genomes in each row are noted in column " $\mathrm{N}$ ". Light blue shading indicates fluoroquinolone resistant genotypes; dark blue shading indicates MSMassociated genotypes. Columns are grouped by typical location of the AMR determinant (labelled horizontal bars at the top): transposon Tn7, represented by marker genes tnSABCDE and class II integron In2 integrase gene intA2; Shigella resistance locus (SRL); spA plasmid, represented by marker gene rep; other mobile elements; mutations in quinolone resistance determining region (QRDR). Column "other Agly" indicates proportion of genomes carrying at $\geq 1$ additional aminoglycoside resistance gene beyond those with their own columns; column 'other' indicates proportion of genomes carrying $\geq 1$ other AMR gene that is not otherwise listed (full AMR gene content per strain is available in Supplementary Data 1).

genes $m p h(\mathrm{~A})$ and $\mathrm{ermB}$ were detected at high frequency in genotypes 3.6.1.1.2 (CipR.MSM5, $n=915,95 \%$ ) and 3.7.29.1.2.1 (VN2.MSM2.Aus, $n=147,90 \%$ ); $m p h(\mathrm{~A})$ was present alone in the single 1.2.1 genome (Vietnam, 2007), and alone or with ermB at lower frequency $(<65 \%)$ amongst other Lineage 3 genotypes (Fig. 3). Notably, $n=947$ 3.6.1.1 (CipR) genomes carried $m p h$ (A) in addition to $\operatorname{Tn} 7$ and $\mathrm{spA}$ genes, rendering them resistant to azithromycin, ciprofloxacin and first-line drugs $(n=58,3.6 .1$; $n=16, \quad 3.6 .1 .1 ; \quad n=868, \quad 3.6 .1 .1 .2 ; \quad n=2,3.6 .1 .1 .3 ; n=3$, 3.6.1.1.3.1), leaving extended-spectrum cephalosporins as the last remaining oral drug. ESBL genes were detected only sporadically, at low frequencies in clades $3.6(13 \%)$ and $3.7(12 \%)$, across 25 distinct genotypes (frequency range, $0.6-100 \%$, median $17.2 \%$ ) (see Fig. 3). Carbapenemase genes were extremely rare, present in only two genomes ( $b l a_{\text {OXA-66 }}$ and $\left.b l a_{\text {OXA-181 }}\right)$. Concerningly, we detected 40 genomes with resistance determinants for azithromycin, third-generation cephalosporins and fluoroquinolones, all within CipR genotype 3.6.1.1 $(n=16,3.6 .1 .1 ; n=11,3.6 .1 .1 .1$; $n=15,3.6 .1 .1 .2)$. These genomes were isolated between 2014 and 2019, and were found in genomes from England $(n=21)$, Australia $(n=13)$, the USA $(n=3)$, Vietnam $(n=3)$ and the Netherlands $(n=2)$ (further details below). 


\section{a Ciprofloxacin resistance}
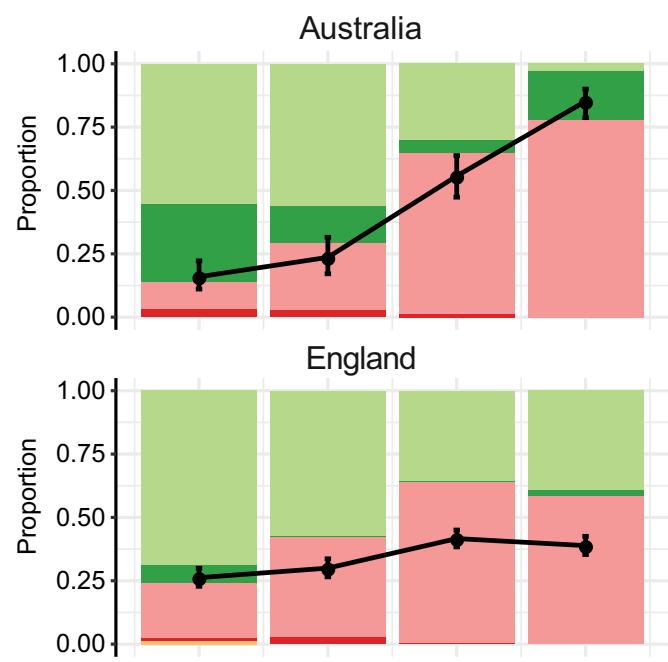

USA

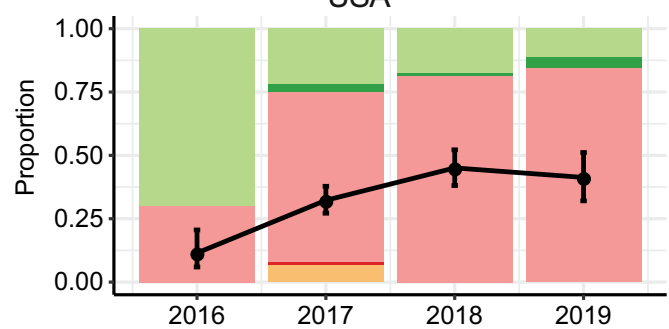

b Azithromycin resistance

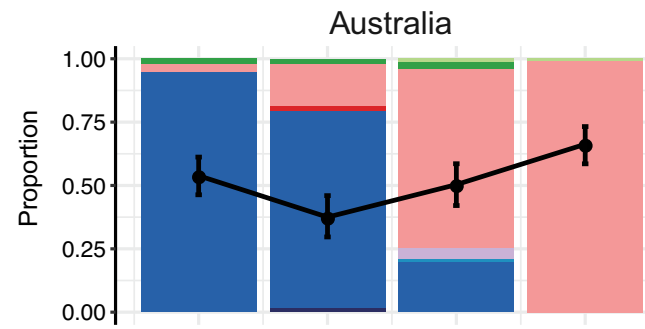

England

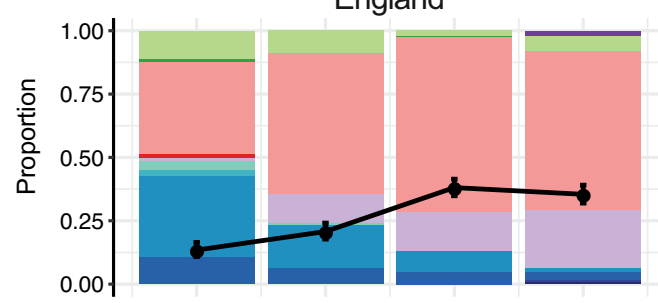

USA

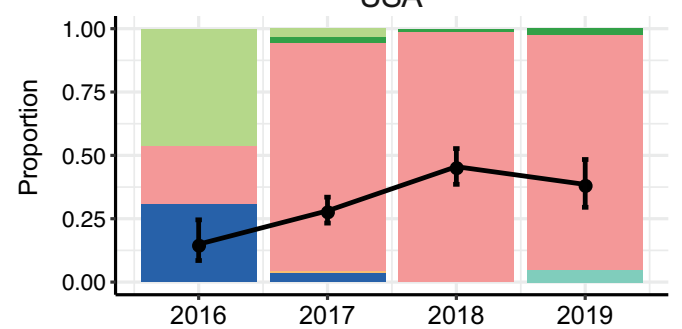

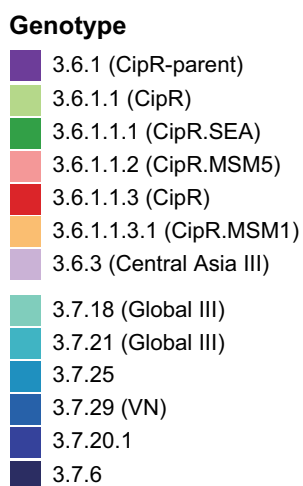

Genotype

3.6.1.1.1 (CipR.SEA)

3.6.1.1.2 (CipR.MSM5)

3.6.1.1.3 (CipR)

3.6.1.1.3.1 (CipR.MSM1)

Central Asia III)

Fig. 4 Prevalence and genotype breakdown of ciprofloxacin and azithromycin resistant S. sonnei in three surveillance regions (Australia, England and USA) from 2016 to 2019. In each plot, black lines indicate the proportion of genomes that are predicted resistant to (a) ciprofloxacin (defined as presence of $\geq 3$ QRDR mutations) or (b) azithromycin (defined as carrying $m p h(A)$ ). Error bars indicate $95 \%$ confidence intervals for the proportion resistant ( $n=$ 144-182 for Australia, $n=610-869$ for England, $n=87-313$ for USA). Stacked bars indicate the relative abundance of each genotype among resistant isolates, coloured by genotype as per legend.

Application to public health surveillance data from Australia, England and USA. To demonstrate how the S. sonnei genotyping framework can facilitate the rapid tracking and reporting of emerging AMR trends across jurisdictions, we applied it to genomic surveillance data from Victoria, Australia $(n=644)$, England $(n=2867)$ and USA $(n=711)$ generated over a 4-year period (2016-2019) (data in Supplementary Data 1). The data represents all cultured isolates submitted to the Microbiological Diagnostic Unit Public Health Laboratory in Australia (42\% of all $S$. sonnei notifications in Victoria $)^{4}$ and all those sent to the Public Health England Gastrointestinal Bacteria Reference Unit ${ }^{28}$ (provided direct from the reference laboratories for the present study); and $\sim 5 \%$ of those notified in the USA (sourced from the public GenomeTrakr database ${ }^{33}$ ). The total time taken to generate genotyping reports (including QRDR mutations) for all $n=$ 4222 isolates was $\sim 40 \mathrm{sec}-1 \mathrm{~min}$ per isolate using Mykrobe, with raw Illumina sequence files (fastq format) as input.

Figure $4 \mathrm{a}$ shows the annual frequency of fluoroquinolone resistance (defined as presence of 3 QRDR mutations) in each country, and the distribution of genotypes amongst the resistant isolates. Increasing fluoroquinolone resistance rates are evident amongst the $S$. sonnei samples from each country, beginning at $\leq 25 \%$ in 2016 and reaching $85 \%$ in Australia, 39\% in England and $44 \%$ in USA in 2019 (black lines, Fig. 4a). All fluroquinolone resistant genomes belonged to genotypes within the CipR sublineage (3.6.1.1), with the subgroup 3.6.1.1.2 (CipR.MSM5) accounting for a steadily increasing proportion of resistant isolates in each country, from $\leq 30 \%$ in 2016 to $78 \%$ in Australia, 59\% in England and 85\% in USA in 2019 (pink bars, Fig. 4a). These results are consistent with local epidemiological outbreaks of $S$. sonnei in MSM communities in England ${ }^{16,17}$ and Australia $^{4,26}$. Notably however, the common nomenclature makes it easy to identify several epidemiologically important patterns: (i) all resistant isolates in all three countries derive from the previously-described CipR sublineage 3.6.1.1 that emerged from South Asia in the early 2000s; (ii) the reported spread of resistant $S$. sonnei in MSM communities in Australia and England involves the same strain (this was not clear from previous reports, as the strain was named "MSM clade 5" in the English studies and formed a subgroup within the "BAPS3 cluster" in the Australian studies); (iii) this strain represents a clonal subgroup of the CipR sublineage (genotype 3.6.1.1.2, CipR.MSM5) that has disseminated intercontinentally over the last few years and become responsible for the majority of fluoroquinolone resistant S. sonnei infections in all three countries. (Note an additional two English isolates of genotype 3.6.1.1 from 2019 carried gyrA-S83L and parC-S80I plus the $q n r S$ gene, which likely combine to confer fluoroquinolone resistance ${ }^{34}$ ).

Figure $4 \mathrm{~b}$ shows the annual frequency of azithromycin resistance (predicted by presence of $m p h(\mathrm{~A})$ ) in each country, and the genotypes responsible. Resistance rates were high $(>50 \%)$ in Australia across the whole period, reflecting documented outbreaks in the MSM community ${ }^{4}$. In England and the USA, rates increased between 2016 and 2019, from 13 to $38 \%$ in England and 15 to $45 \%$ in the USA (black lines, Fig. $4 \mathrm{~b}$ ). The genotype distributions amongst $m p h(\mathrm{~A})+$ genomes differed markedly between countries in 2016, dominated by 3.7.29 (VNclone, 95\%) in Australia, 3.7.25 (MSM4, 32\%) and 3.6.1.1.2 (CipR.MSM5, 36\%) in England, and 3.6.1.1 (CipR, 46\%), 3.7.29 (VNclone, 31\%) and 3.6.1.1.2 (CipR.MSM5, 23\%) in the USA (see 


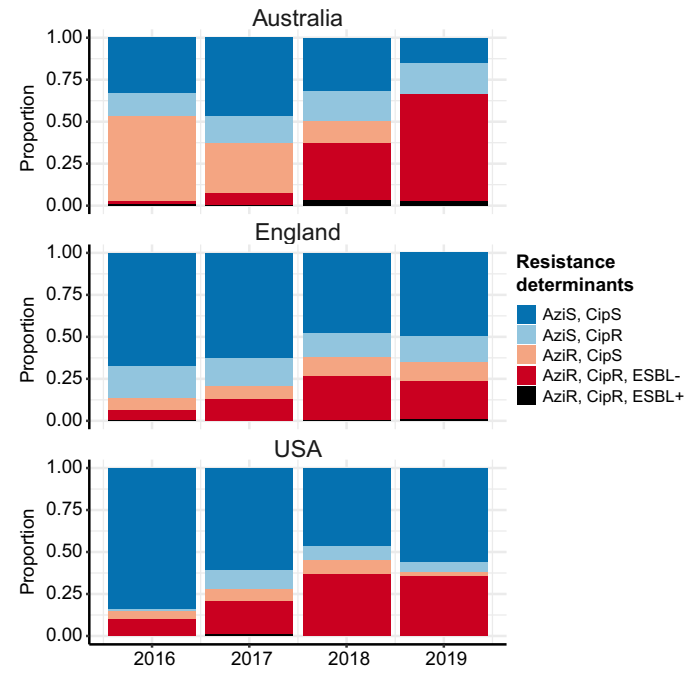

Fig. 5 Prevalence of combined resistance to ciprofloxacin and azithromycin amongst genomes from each surveillance region. Stacked bar colours indicate the relative abundance of different combinations of resistances, predicted from genomes: $\mathrm{CipR}$, ciprofloxacin resistant (defined as presence of $\geq 3$ QRDR mutations); AziR, azithromycin resistant (defined as carrying $m p h(A)$ ); ESBL+, presence of extended-spectrum beta-lactamase (ESBL) gene associated with resistance to third generation cephalosporins (bla $\left.a_{\mathrm{CTX}-\mathrm{M}-14}, b l a_{\mathrm{CTX}-\mathrm{M}-15}, b / a_{\mathrm{CTX}-\mathrm{M}-27}, b l a_{\mathrm{CTX}-\mathrm{M}-55}, b / a_{\mathrm{CTX}-\mathrm{M}-134}\right)$.

barplot, Fig. 4b). Notably though, the contribution of 3.6.1.1.2 (CipR.MSM5) increased dramatically in each country, and in 2019 accounted for $99 \%$ of $m p h(A)+$ isolates in Australia, $62 \%$ in England and $93 \%$ in USA (bars, Fig. 4b). Thus in 2019, the majority of predicted azithromycin resistant isolates in all three countries were also predicted to be resistant to ciprofloxacin and first line drugs. Concerningly, the proportion of total S. sonnei genomes with combined resistance determinants for azithromycin, ciprofloxacin and first-line drugs was high (36-66\%) in all three countries in 2019 (Fig. 5).

Whilst ESBL genes were rare across the $S$. sonnei surveillance data $(7.8 \%$ in Australia, $17.5 \%$ in England, $1 \%$ in USA, see Supplementary Data 1), concerningly 201 genomes carried ESBL genes in addition to $m p h(\mathrm{~A})$, and 36 of these were QRDR triple mutants belonging to 3.6.1.1 (CipR). The latter were concentrated in genotypes 3.6.1.1, 3.6.1.1.1 and 3.6.1.1.2 and comprised 13 Australian genomes (2\% of total, 2016-2019, all three genotypes), 20 English genomes $(0.7 \%$ of total, in 2016 and 2018-2019, all three genotypes) and 3 USA genomes (0.4\% of total, 2017 , only 3.6.1.1 and 3.6.1.1.1) (Fig. 5). Consistent with the overall picture of ESBL genes in S. sonnei described above (Fig. 3), there was no evidence of strong linkage between specific ESBL genes and particular genotype backgrounds in the public health surveillance data for this period (Supplementary Data 1). This was true even amongst CipR/AziR/ESBL + genomes, which included eight unique combinations of genotype and $b a_{\mathrm{CTX}-\mathrm{M}}$ allele (3-6 per country, Supplementary Fig. 6), consistent with multiple independent acquisitions of different AMR plasmids in different settings contributing to the march towards pan-resistance to oral drugs.

\section{Discussion}

Here, we provide a global framework for S. sonnei, and identify marker SNVs that can be used to easily position newly sequenced isolates into this framework, without the need for timeconsuming comparative genomics or phylogenetic analysis. We demonstrate that the population structure of $S$. sonnei can be represented by a robust maximum likelihood phylogeny and define within it 137 subtrees on the basis of pairwise divergence and epidemiological coherence, which we designate as hierarchically nested genotypes. Furthermore, we provide a software package implemented within the Mykrobe ${ }^{32}$ code base, which can identify both the $S$. sonnei genotype and QRDR SNVs direct from short-read sequence files in a few seconds.

The genotyping scheme was constructed with a view towards stability, prioritising as markers SNVs that are found in highly conserved core genes that are not under adaptive selection in the population. We also endeavoured to make the scheme backwards compatible by identifying and designating unique genotypes for $S$. sonnei genetic clusters defined in previous studies on the basis of epidemiological features (see Table 2). We further aimed to ensure genotypes are interpretable, with stable numerical identifiers that convey relationships between genotypes, and human readable aliases that convey relevant epidemiological information where appropriate (see Table 2). The genotyping scheme can be readily expanded in future, by adding new genotypes and corresponding SNV markers as new clusters of agreed epidemiological importance are identified based on phylogenetic analyses. We envisage managing future updates via an international working group consisting of epidemiological, public health and genomics experts who utilise the scheme, similar to the approach proposed for a recent Neisseria gonorrhoeae typing scheme ${ }^{35}$, and have adopted clear versioning to facilitate compatibility between laboratories and over time. Importantly, our approach provides genotype definitions and nomenclature that are stable and transparent, not dependent on comparative analysis or on any specific sequencing assay or software package, although we provide an implementation in the Mykrobe v0.9.0 software for convenience. The Mykrobe implementation was tested on all Illumina reads used in this study, and matching long (ONT) reads for eight of these genomes. Correct genotypes were returned in all cases (providing the --ont flag to Mykrobe when using the long reads as input). This overall approach is designed to facilitate easy communication between laboratories and jurisdictions, and straightforward comparison of pathogen populations over time, allowing for rapid identification of clonal dissemination and AMR trends.

To demonstrate the utility of the genotyping scheme for public health applications, we applied it to summarise large genomic surveillance datasets from three jurisdictions in Australia, England and the USA. The results provide a straightforward view of temporal trends in the populations of S. sonnei causing disease in each jurisdiction. They also clearly identify common ciprofloxacin and azithromycin resistant clones that have spread globally and are now present in all three jurisdictions, which was previously not obvious from individual studies, as there was no common nomenclature.

The genotyping approach introduced here could greatly simplify the bioinformatics procedures required for routine genomic surveillance of $S$. sonnei in reference laboratories, the first step of which usually involves comparison of newly sequenced genomes to those from prior cases in the same jurisdiction (to monitor local trends) and/or other jurisdictions (to monitor introduction of new strains and patterns of regional spread). Notably, the public health labs in Australia, England and USA from which we sourced genomic surveillance data (Fig. 4) all utilise genome-wide SNV-based phylogenetics for S. sonnei analysis ${ }^{17,26}$.

Reliance on whole genome comparisons and phylogenetic inference is considerably slower than genotyping and requires expertise and background knowledge both to conduct the analysis and to interpret the results. In contrast, using the genotyping framework, raw sequence data can be turned into simple informative identifiers for each strain without reference to other 
genomes or databases (and without needing to assemble the genome). The resulting genotyping information can be easily interpreted, compared and stored in (non-sequence) databases for future reference, facilitating epidemiological investigations without need for direct comparisons with any other genome sequences. Detailed phylogenetic analysis can then be applied to subsets of isolates that share the same or similar genotypes, if needed to address specific questions (e.g., relating to emerging local outbreaks or transmission networks). Notably, such phylogenetic analyses are essential to identify the emergence of novel clusters from within currently defined genotypes. If a high-quality $S$. sonnei genome sequence lacked all the lineage-specific SNV markers in the genotyping scheme, this would suggest it belongs to a novel lineage. Such cases could be investigated further using phylogenomics, and the scheme expanded to include the novel lineage. While this is expected to be rare, it may well occur as more sequencing is undertaken in Africa and other regions currently under-represented by the available genomic data.

For example, scientists investigating azithromycin-resistant $S$. sonnei isolated from MSM in Switzerland recently reported identifying the strains as belonging to the same clones spreading through MSM communities in England. To achieve this identification, they had to download English genome data, compare their newly isolated genomes to these using read mapping, and construct and interpret whole genome phylogenies ${ }^{29}$. A similar study using WGS to investigate a ciprofloxacin-resistant outbreak in California used the same informatics approach to conclude that the local strain belonged to the previously described ciprofloxacin resistant lineage originating in South Asia ${ }^{23}$. Another recent study from Switzerland used WGS to investigate an increase in ESBL S. sonnei using a combination of cgMLST and phylogenetics ${ }^{36}$. Using our new genotyping approach, all of these identifications could be made within minutes of obtaining sequence data, with no need for external comparative data, background knowledge of $S$. sonnei genetics, or complex computational infrastructure and expertise (see Supplementary Note 1, Supplementary Fig. 7, Supplementary Table 3 and Supplementary Data 3). The rapid identification of genomicallyrelated isolates via genotyping could be used to facilitate timely public health responses to shigellosis outbreaks.

A potential alternative to SNV-based genotyping is cgMLST, available for Shigella/E. coli via EnteroBase ${ }^{9}$. The scheme consists of 2513 genes as markers ( half the genome), including loci under positive selection such as gyrA and parC, which are used to define core genome sequence types (cgSTs). EnteroBase also assigns cgSTs to hierarchical clusters (HC) with stable names, using the HierCC algorithm ${ }^{9}$, at various locus-distance thresholds $(2,5,10,20$, etc). HC5 is widely used for detecting outbreak clusters of Salmonella and E. coli in foodborne surveillance ${ }^{37-41}$, however clustering of S. sonnei at HC5 (or even HC10) breaks apart epidemiologically recognised outbreak clusters such as the MSM clades 3.6.1.1.3.1 (CipR.MSM1), 3.6.1.1.2 (CipR.MSM5) and 3.7.25 (MSM4) (see Supplementary Fig. 8), making them hard to track. We speculate that the over-division of S. sonnei by cgMLST may be due to the fact that many alleles are affected by deletion or insertion sequence activity, as is common in $S$. sonne $i^{42}$. Notably our focused genotyping scheme is designed to utilise a small number of highly stable loci, avoiding this issue. In addition, cgST numbers and HierCC cluster numbers are arbitrarily assigned and carry no meaning beyond being unique identifiers, whereas hierarchical naming systems such as those used in our scheme (and those adopted for M. tuberculosis, S. Typhi and SARS-CoV-2 ${ }^{43}$ schemes) are much more informative as they communicate relationships between clusters. Perhaps for these reasons, there is to-date only one published report of $S$. sonnei analysis that utilised cgMLST for public health investigation $^{36}$, and they too relied on additional SNV-based phylogenetics to place their local isolates in the context of global populations as this was not easily ascertained using cgMLST (see Example 2 in Supplementary Note 1, Supplementary Data 3).

In conclusion, while genomics is increasingly becoming a standard tool for surveillance of $S$. sonnei and other pathogens in public health reference laboratories, this poses computational and epidemiological challenges in terms of analysis, interpretation and communication of genome-derived data across discipline and jurisdictional boundaries. The genotyping framework and universal nomenclature for $S$. sonnei established here provides a solution for many of these issues, and provides a structure to enable clear communication between public health and basic science research groups. Importantly, it will facilitate monitoring of the emergence and spread of AMR S. sonnei clones, at local and global levels, which will become increasingly important as public health agencies face the emerging threat of pan-resistant $S$. sonnei.

\section{Methods}

Single nucleotide variants (SNVs) and phylogenetic analysis - discovery dataset. The discovery dataset consisted of 1935 high quality previously published S. sonnei genomes, sequenced using Illumina platforms. Source information for all genomes (year of collection, geographic origin, etc) was extracted from their respective publications $s^{4,7,10-13,15,16}$ (Supplementary Table 1 and Supplementary Data 1). Genomes were mapped to the $S$. sonnei reference genome $53 \mathrm{G}$ (accession NC_016822) using RedDog (v1beta11; https://github.com/katholt/RedDog). Briefly, RedDog maps reads with Bowtie2 v2.2.944 with the sensitive local parameter, then uses SAMtools $v 1.1^{45}$ to retain high quality SNV calls (phred score $\geq 20$, read depth $>5$, removes heterozygous calls). SNVs detected in repetitive regions where SNV calls are dubious (e.g. insertion sequences, phage) were removed (see Figshare, https://doi.org/10.26180/5f1a443b19b2f). SNVs associated with recombination were identified and excluded using Gubbins v2.3.2 ${ }^{46}$ ( $n=208$ SNVs across 10 genomes). The final alignment consisted of 23,673 SNVs across 1935 genomes. Ancestral alleles at these SNV sites were extracted from five E. coli genomes (accessions CP019005, CP031916, CP019961, CP034399 and CP019259) using the mapping procedure described above, and were included in the alignment for the purpose of outgroup rooting the tree. A maximum likelihood (ML) phylogeny was inferred using IQ-TREE v2 ${ }^{47}$ using a GTR substitution model (Fig. 1b). An interactive form of the tree is available in Microreact, at https://microreact.org/project/ fG2N7huk9oZNCaVHu8rukr.

Defining clades and subclades of the genotyping scheme. The discovery set was analysed to define appropriate SNV thresholds for assigning genomes to genotypes based on pairwise genetic distances. Pairwise SNV distances were calculated for all pairs of, and thresholds were selected by examining the distribution of pairwise SNV distances (Fig. 2a), to define clusters at three levels: lineage (600 SNVs), clade (215 SNVs) and subclade (100 SNVs). To cluster discovery set genomes at these thresholds, we applied hierarchical clustering (using hclust function in $\mathrm{R}$, with complete linkage) to the pairwise SNV distance matrix. The resulting dendrogram was then cut at the aforementioned thresholds (using the $\mathrm{R}$ function cutree) to cluster isolates into discrete groups representing lineages, clades and subclades. The resulting groups were compared to the ML phylogeny to check that each was monophyletic (using the function is.monophyletic in the ape package ${ }^{48}$ for R); a small number of groups were non-monophyletic and were broken up into smaller groups ( 2 groups at clade level, $n=388$ isolates; 3 groups at subclade level, $n=52$ isolates) to result in a final set of monophyletic clusters. The SNV alignment was also analysed using the $\mathrm{R}$ package fastbaps ${ }^{49}$ to partition the data into groups using Bayesian clustering (using the $h c$ method for prior optimisation, and two levels of clustering using multi_res_baps) (Supplementary Fig. 1a). Pairwise SNV distances within and between our final genotype groups (Fig. 2b), and within and between FastBAPS clusters for comparison (Supplementary Fig. 1b), were calculated from the SNV alignments using the dna.dist function in the ape package ${ }^{48}$. Isolates belonging to previously defined epidemiological groups were located in the ML tree, and used to identify subtrees corresponding to each set of group members. For epidemiological groups defined on the basis of AMR determinants, the presence of these determinants (identified using SRST2 ${ }^{50}$ and the QRDR SNVs) was used to determine the boundaries of the local subtree sharing the defining features of the group (Table 2).

Developing and implementing SNV-based genotyping scheme. We identified potential marker SNVs for each of the 147 genotypes by mapping SNVs onto the branches of the ML phylogeny using SNPPar ${ }^{51}$ with default parameters. A total of 980 homoplasic SNVs were identified and excluded from consideration as markers. For most genotypes multiple markers mapped to the defining branch; we prioritised synonymous changes within core genes $(n=138)$ over nonsynonymous $(n=$ 
8) or intergenic SNVs $(n=1)$, and where multiple synonymous SNVs were available we prioritised genes with the lowest ratio of nonsynonymous:synonymous SNVs (calculated from SNPPar output) as these are less likely to be under selection in the population and thus serve as stable marker SNVs. The list of SNV markers is given in Supplementary Data 2.

We modified the Mykrobe genotyping software ${ }^{32}$ to probe for these marker SNVs and assign S. sonnei genotypes. Probes to detect changes at specific S. sonnei QRDR codons (GyrA-83, GyrA-87, ParC-80) were also included in the S. sonnei

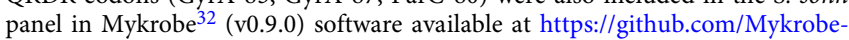
tools/mykrobe, using the probe panel stored in Figshare (https://doi.org/10.6084/ m9.figshare.13072646). Mykrobe outputs were then parsed using a custom Python script which tabulates results across a set of samples and summarises the support for each genotype call (https://github.com/katholt/sonneityping). We tested the Mykrobe $S$. sonnei genotyper using the discovery genome set as input (Illumina reads, fastq format), to confirm that the genotype and QRDR mutations reported by this implementation were correct. Code was also tested using Oxford Nanopore (ONT) long reads (fastq format) using the --ont flag in Mykrobe.

Validating the genotyping scheme on independent data. We used an independent validation dataset (i.e., genomes not included in the discovery set used to define the scheme) a total of 2015 S. sonnei genomes downloaded from the GenomeTrakr project $^{33}$ on 7 May 72019 (listed in Supplementary Data 1). All genomes had been sequenced using Illumina platforms. The validation and discovery datasets were subjected to mapping, SNV calling and phylogenetic analysis as described above, generating a recombination-filtered core-genome alignment of 32,138 SNVs in 3696 isolates $(n=260$ SNVs identified as recombinant across 11 genomes), and a ML phylogeny (Supplementary Fig. 3, interactive tree available in Microreact at https://microreact.org/project/g8BvA2JCXWaZNDyPyjsWXF). All genomes were assigned a genotype using Mykrobe ${ }^{32}$ v0.9.0, and these were compared to the ML phylogeny to check that each genotype was monophyletic as expected (using the function is.monophyletic in the ape package ${ }^{48}$ for R). In addition, we confirmed that all marker SNVs remained non-homoplasic amongst this larger set of genomes, using output from SNPPar (a total of 2003 homoplastic SNVs were identified amongst this expanded set of genomes).

Analysis of AMR determinants amongst genotypes. Genotypes were identified as above, and AMR determinants were identified using SRST2 ${ }^{50}$ and the CARD database ${ }^{52}$ for a further 2644 genomes sourced from public databases, and results combined with those from the discovery and validation sets yielding a total of $n=$ 6595 S. sonnei genomes for analysis. Genotypes with at least ten representatives in this data set (total $n=57$ genotypes), and AMR genes detected in at least two genomes, were included in the analysis of AMR frequencies within genotypes (Fig. 3 and Supplementary Fig. 4). Data were analysed in R and visualised using the pheatmap and ggridges packages.

To assess the correspondence between genotypes defined here within the CipR clade (genotype 3.6.1.1 and its subtypes) and the two subpopulations (Pop1, Pop2) defined previously by The et al. ${ }^{14}$, we constructed a ML phylogeny including all genomes analysed in The et al. ${ }^{14}$ and at least one representative from each Lineage 3 clade and each genotype in clade 3.6. Genomes which had not already been included in the discovery set were genotyped, and subjected to mapping and SNV calling for inclusion in phylogenetic analysis as described above, generating an alignment of 9178 SNVs and an ML phylogeny (Supplementary Fig. 5, interactive tree available in Microreact at https://microreact.org/project/kMRoFFXxkB6JAn9bgBAdMz).

Application to public health surveillance data. We applied the S. sonnei genotyping framework to analyse 4222 genomes sequenced by public health reference labs between 2016 and 2019 in Australia $\left(n=644^{4}\right)$, England $\left(n=2867^{16,17}\right)$, and the USA ( $n=711$, from GenomeTrakr ${ }^{33}$ as of 7 May 2019). All genomes were genotyped, and AMR determinants were identified as above. Azithromycin resistance was predicted based on presence of $m p h(\mathrm{~A}), \mathrm{ESBL} / \mathrm{carb}$ apenemase production based on known beta-lactamase alleles (CTX-M, OXA-66, OXA-181), and ciprofloxacin resistance based on the combination of three QRDR mutations (GyrA-S83L, GyrA-D87Y and ParC-S80I). Data were analysed in R and visualised using the ggplot2 package (Figs. 4-5).

Reporting summary. Further information on research design is available in the Nature Research Reporting Summary linked to this article.

\section{Data availability}

Supplementary Data 1 lists all genome data used, with read accessions, source information, genotype calls and AMR determinants

Marker SNVs used to define genotypes are tabulated in Supplementary Data 2 and GitHub, https://doi.org/10.5281/zenodo.4609813; and in Mykrobe panel format in Figshare, https://doi.org/10.6084/m9.figshare.13072646

AMR genes were detected from CARD database v3.0.8, available at https://card. mcmaster.ca/download

Regions of the S. sonnei $53 \mathrm{G}$ reference genome excluded from SNV calling are available in Figshare, https://doi.org/10.26180/5f1a443b19b2f
Interactive annotated trees are available in Microreact for the following collections: Discovery data: https://microreact.org/project/fG2N7huk9oZNCaVHu8rukr Validation data: https://microreact.org/project/g8BvA2JCXWaZNDyPyjsWXF CipR clade: https://microreact.org/project/kMRoFFXxkB6JAn9bgBAdMz

\section{Code availability}

Mykrobe v0.9.0 is available for download at https:/github.com/Mykrobe-tools/mykrobe

Instructions for running Mykrobe v0.9.0 for S. sonnei and tabulating the results is available at https://github.com/katholt/sonneityping (https://doi.org/10.5281/ zenodo.4609813)

Received: 29 October 2020; Accepted: 23 March 2021; Published online: 11 May 2021

\section{References}

1. Kotloff, K. L., Riddle, M. S., Platts-Mills, J. A., Pavlinac, P. \& Zaidi, A. K. M. M. Shigellosis. Lancet 391, 801-812 (2018).

2. Livio, S. et al. Shigella isolates from the global enteric multicenter study inform vaccine development. Clin. Infect. Dis. 59, 933-941 (2014).

3. Baker, K. S. et al. Intercontinental dissemination of azithromycin-resistant shigellosis through sexual transmission: a cross-sectional study. Lancet Infect. Dis. 15, 913-921 (2015)

4. Ingle, D. J. et al. Co-circulation of multidrug-resistant shigella among men who have sex with men in Australia. Clin. Infect. Dis. 69, 1535-1544 (2019).

5. Kotloff, K. L., Winickoff, J. P. \& Ivanoff, B. Global burden of Shigella infections: implications for vaccine development and implementation of control strategies. Bull. World Health Organ 77, 666 (1999).

6. Vinh, H. et al. A changing picture of shigellosis in southern Vietnam: shifting species dominance, antimicrobial susceptibility and clinical presentation. BMC Infect. Dis. 9, 204 (2009).

7. Holt, K. E. et al. Shigella sonnei genome sequencing and phylogenetic analysis indicate recent global dissemination from Europe. Nat. Genet. 44, 1056-1059 (2012).

8. McDonnell, J. et al. Retrospective analysis of whole genome sequencing compared to prospective typing data in further informing the epidemiological investigation of an outbreak of Shigella sonnei in the UK. Epidemiol. Infect. https://doi.org/10.1017/S0950268813000137 (2013)

9. Zhou, Z., Alikhan, N. F., Mohamed, K., Fan, Y. \& Achtman, M. The EnteroBase user's guide, with case studies on Salmonella transmissions, Yersinia pestis phylogeny, and Escherichia core genomic diversity. Genome Res. https://doi.org/10.1101/gr.251678.119 (2020).

10. Baker, K. S. et al. Whole genome sequencing of Shigella sonnei through PulseNet Latin America and Caribbean: advancing global surveillance of foodborne illnesses. Clin. Microbiol. Infect. 23, 845-853 (2017).

11. Holt, K. E. et al. Tracking the establishment of local endemic populations of an emergent enteric pathogen. Proc. Natl Acad. Sci. USA. 110, 17522-17527 (2013).

12. The, H. C. et al. Introduction and establishment of fluoroquinolone-resistant Shigella sonnei into Bhutan. Microb. Genomics 1, e000042 (2015).

13. The, H. C. et al. South Asia as a reservoir for the global spread of ciprofloxacin-resistant Shigella sonnei: a cross-sectional study. PLoS Med. 13, e1002055 (2016).

14. The, H. C. et al. Dissecting the molecular evolution of fluoroquinoloneresistant Shigella sonnei. Nat. Commun. 10, 4828 (2019).

15. Baker, K. S. et al. Travel- and community-based transmission of multidrugresistant Shigella sonnei lineage among international Orthodox Jewish communities. Emerg. Infect. Dis. 22, 1545-1553 (2016).

16. Baker, K. S. et al. Genomic epidemiology of Shigella in the United Kingdom shows transmission of pathogen sublineages and determinants of antimicrobial resistance. Sci. Rep. 8, 7389 (2018).

17. Bardsley, M. et al. Persistent transmission of shigellosis in England is associated with a recently emerged multidrug-resistant strain of Shigella sonnei. J. Clin. Microbiol. 58, e01692-19 (2020).

18. Baker, K. S. et al. Horizontal antimicrobial resistance transfer drives epidemics of multiple Shigella species. Nat. Commun. 9, 1462 (2018).

19. Ward, D. V. et al. Integration of genomic and clinical data augments surveillance of healthcare-acquired infections. Infect. Control Hosp. Epidemiol. 40, 649-655 (2019)

20. Yang, F. et al. Genome dynamics and diversity of Shigella species, the etiologic agents of bacillary dysentery. Nucleic Acids Res 33, 6445-6458 (2005).

21. McIver, C. J. et al. Epidemic strains of Shigella sonnei biotype g carrying integrons. J. Clin. Microbiol. https://doi.org/10.1128/JCM.40.4.1538-1540.2002 (2002). 
22. Turner, S. A., Luck, S. N., Sakellaris, H., Rajakumar, K. \& Adler, B. Molecular epidemiology of the SRL pathogenicity island. Antimicrob. Agents Chemother. 47, 727-734 (2003).

23. Kozyreva, V. K. et al. Recent outbreaks of Shigellosis in California caused by two distinct populations of Shigella sonnei with either increased virulence or fluoroquinolone resistance. mSphere 1, 1-18 (2016).

24. Mook, P. et al. ESBL-producing and macrolide-resistant Shigella sonnei infections among men who have sex with men, England, 2015. Emerg. Infect. Dis. 22, 1948-1952 (2016).

25. Rew, V. et al. Whole-genome sequencing revealed concurrent outbreaks of shigellosis in the English orthodox Jewish community caused by multiple importations of Shigella sonnei from Israel. Microb. Genomics 4, e000170 (2018).

26. Williamson, D., Ingle, D. \& Howden, B. Extensively drug-resistant shigellosis in australia among men who have sex with men. N. Engl. J. Med. 381, 2477-2479 (2019).

27. Abelman, R. L., M'ikanatha, N. M., Figler, H. M. \& Dudley, E. G. Use of whole genome sequencing in surveillance for antimicrobial-resistant Shigella sonnei infections acquired from domestic and international sources. Microb. Genomics 5, e000270 (2019).

28. Sadouki, Z. et al. Comparison of phenotypic and WGS-derived antimicrobial resistance profiles of Shigella sonnei isolated from cases of diarrhoeal disease in England and Wales, 2015. J. Antimicrob. Chemother. 72, 2496-2502 (2017).

29. Vladimira, H., Helena, S. S., Marcel, S., Daniel, G. \& Adrian, E. First report of sexually transmitted multi-drug resistant Shigella sonnei infections in Switzerland, investigated by whole genome sequencing. Swiss Med. Wkly. 148, 14645 (2018).

30. Coll, F. et al. A robust SNP barcode for typing Mycobacterium tuberculosis complex strains. Nat. Commun. 5, 4812 (2014).

31. Wong, V. K. et al. An extended genotyping framework for Salmonella enterica serovar Typhi, the cause of human typhoid. Nat. Commun. 7, 12827 (2016).

32. Hunt, M. et al. Antibiotic resistance prediction for Mycobacterium tuberculosis from genome sequence data with Mykrobe. Wellcome Open Res. https://doi.org/10.12688/wellcomeopenres.15603.1 (2019).

33. Jackson, B. R. et al. Implementation of Nationwide Real-time Whole-genome Sequencing to Enhance Listeriosis Outbreak Detection and Investigation. Clin. Infect. Dis. 63, 380-386 (2016).

34. Neuert, S. et al. Prediction of phenotypic antimicrobial resistance profiles from whole genome sequences of non-typhoidal Salmonella enterica. Front. Microbiol. 9, 592 (2018).

35. Sánchez-Busó, L. et al. A community-driven resource for genomic surveillance of Neisseria gonorrhoeae at Pathogenwatch. bioRxiv https://doi.org/10.1101/ 2020.07.03.186726 (2020)

36. Campos-Madueno, E. I. et al. Rapid Increase of CTX-M-Producing Shigella sonnei Isolates in Switzerland: Spread of Common Plasmids and International Clones. Antimicrob. Agents Chemother. https://doi.org/10.1128/aac.01057-20 (2020).

37. Nouws, S. et al. The benefits of whole genome sequencing for foodborne outbreak investigation from the perspective of a national reference laboratory in a smaller country. Foods https://doi.org/10.3390/foods9081030 (2020).

38. Smith, A. M. et al. Whole-genome sequencing to investigate two concurrent outbreaks of salmonella enteritidis in South Africa, 2018. J. Med. Microbiol. https://doi.org/10.1099/jmm.0.001255 (2020).

39. Robinson, E. et al. Outbreak of Salmonella Newport associated with internationally distributed raw goats' milk cheese, France, 2018. Epidemiol. Infect. https://doi.org/10.1017/S0950268820000904 (2020).

40. Jones, G. et al. Outbreak of Salmonella enterica serotype Poona in infants linked to persistent Salmonella contamination in an infant formula manufacturing facility, France, August 2018 to February 2019. Eurosurveillance https://doi.org/10.2807/1560-7917.ES.2019.24.13.1900161 (2019).

41. Jones, G. et al. Outbreak of shiga toxin-producing Escherichia coli (STEC) O26 paediatric haemolytic uraemic syndrome (HUS) cases associated with the consumption of soft raw cow's milk cheeses, France, March to May 2019. Eurosurveillance https://doi.org/10.2807/1560-7917.ES.2019.24.22.1900305 (2019).

42. Hawkey, J., Monk, J. M., Billman-Jacobe, H., Palsson, B. \& Holt, K. E. Impact of insertion sequences on convergent evolution of Shigella species. PLoS Genet. https://doi.org/10.1371/journal.pgen.1008931 (2020).

43. Rambaut, A. et al. A dynamic nomenclature proposal for SARS-CoV-2 lineages to assist genomic epidemiology. Nat. Microbiol. https://doi.org/ 10.1038/s41564-020-0770-5 (2020).

44. Langmead, B. \& Salzberg, S. L. Fast gapped-read alignment with Bowtie 2. Nat. Methods 9, 357-359 (2012).

45. $\mathrm{Li}, \mathrm{H}$. et al. The sequence alignment/map format and SAMtools. Bioinformatics 25, 2078-2079 (2009).

46. Croucher, N. J. et al. Diversification of bacterial genome content through distinct mechanisms over different timescales. Nat. Commun. 5, 5471.
47. Minh, B. Q. et al. IQ-TREE 2: New models and efficient methods for phylogenetic inference in the genomic era. Mol. Biol. Evol. (2020) https://doi. org/10.1093/molbev/msaa015PMID - 32011700.

48. Paradis, E. \& Schliep, K. Ape 5.0: An environment for modern phylogenetics and evolutionary analyses in R. Bioinformatics https://doi.org/10.1093/ bioinformatics/bty633 (2019).

49. Tonkin-Hill, G., Lees, J. A., Bentley, S. D., Frost, S. D. W. \& Corander, J. Fast hierarchical Bayesian analysis of population structure. Nucleic Acids Res. 47, 5539-5549 (2019).

50. Inouye, $M$. et al. SRST2: rapid genomic surveillance for public health and hospital microbiology labs. Genome Med 6, 90 (2014).

51. Edwards, D. J., Duchêne, S., Pope, B. \& Holt, K. E. SNPPar: identifying convergent evolution and other homoplasies from microbial whole-genome alignments. bioRxiv 2020.07.08.194480 https://doi.org/10.1101/ 2020.07.08.194480 (2020).

52. Alcock, B. P. et al. CARD 2020: antibiotic resistome surveillance with the comprehensive antibiotic resistance database. Nucleic Acids Res. https://oi. org/10.1093/nar/gkz935 (2020).

\section{Acknowledgements}

K.E.H. is supported by a Senior Medical Research Fellowship from the Viertel Foundation of Australia, and Bill and Melinda Gates Foundation, Seattle (grant \#OPP1175797). K.S.B. is supported by a Wellcome Trust Clinical Research Career Development Fellowship (A/106690/A/14/Z) and RJB through a UKRI MRC New Investigator Research Grant (held by KSB, MR/R020787/1). K.S.B., T.J.D. and C.J. are affiliated to the National Institute for Health Research Health Protection Research Unit (NIHR HPRU) in Gastrointestinal Infections at University of Liverpool in partnership with Public Health England (PHE), in collaboration with University of Warwick. The views expressed are those of the author(s) and not necessarily those of the NHS, the NIHR, the Department of Health and Social Care or Public Health England. D.A.W. is supported by an Investigator Grant from the National Health and Medical Research Council (NHMRC) of Australia (APP1174555).

\section{Author contributions}

Conceptualization, J.H. and K.E.H.; Methodology, J.H., K.P. and K.E.H.; Software, J.H., K.P., L.C., Z.I., M.H., and K.E.H.; Validation, J.H., K.P., K.S.B, R.J.B., L.C., D.J.I, D.A.W and K.E.H.; Formal analysis, J.H., K.P. and K.E.H., Data Curation, K.P., K.S.B., F-X.W., N.R.T, S.B., T.J.D., C.J. and D.A.W., Writing - Original Draft, J.H., K.P. and K.E.H., Writing - Review \& Editing, all authors; Visualization, J.H., K.P., and K.E.H.; Supervision, J.H. and K.E.H.; Project administration, K.E.H; Funding acquisition, K.E.H.

\section{Competing interests}

The authors declare no competing interests.

\section{Additional information}

Supplementary information The online version contains supplementary material available at https://doi.org/10.1038/s41467-021-22700-4.

Correspondence and requests for materials should be addressed to J.H.

Peer review information Nature Communications thanks the anonymous reviewers for their contribution to the peer review of this work. Peer review reports are available.

Reprints and permission information is available at http://www.nature.com/reprints

Publisher's note Springer Nature remains neutral with regard to jurisdictional claims in published maps and institutional affiliations.

\section{(i)}

Open Access This article is licensed under a Creative Commons Attribution 4.0 International License, which permits use, sharing, adaptation, distribution and reproduction in any medium or format, as long as you give appropriate credit to the original author(s) and the source, provide a link to the Creative Commons license, and indicate if changes were made. The images or other third party material in this article are included in the article's Creative Commons license, unless indicated otherwise in a credit line to the material. If material is not included in the article's Creative Commons license and your intended use is not permitted by statutory regulation or exceeds the permitted use, you will need to obtain permission directly from the copyright holder. To view a copy of this license, visit http://creativecommons.org/ licenses/by/4.0/.

(C) The Author(s) 2021 


\section{University Library}

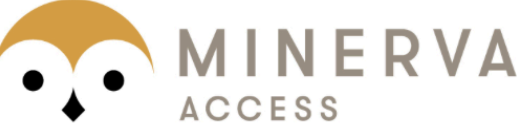

A gateway to Melbourne's research publications

Minerva Access is the Institutional Repository of The University of Melbourne

\section{Author/s:}

Hawkey, J;Paranagama, K;Baker, KS;Bengtsson, RJ;Weill, F-X;Thomson, NR;Baker, S;Cerdeira, L;lqbal, Z;Hunt, M;Ingle, DJ;Dallman, TJ;Jenkins, C;Williamson, DA;Holt, KE

Title:

Global population structure and genotyping framework for genomic surveillance of the major dysentery pathogen, Shigella sonnei

Date:

2021-05-11

\section{Citation:}

Hawkey, J., Paranagama, K., Baker, K. S., Bengtsson, R. J., Weill, F. -X., Thomson, N. R., Baker, S., Cerdeira, L., Iqbal, Z., Hunt, M., Ingle, D. J., Dallman, T. J., Jenkins, C., Williamson, D. A. \& Holt, K. E. (2021). Global population structure and genotyping framework for genomic surveillance of the major dysentery pathogen, Shigella sonnei. NATURE COMMUNICATIONS, 12 (1), https://doi.org/10.1038/s41467-021-22700-4.

Persistent Link:

http://hdl.handle.net/11343/278243

License:

CC BY 